\title{
Systematic Literature Review: Stock Price Prediction Using Machine Learning and Deep Learning
}

\author{
Grace Yoby Dopi ${ }^{1, *}$, Rudy Hartanto ${ }^{2}$, Silmy Fauziati $^{3}$ \\ ${ }^{1}$ Department of Information Technology, Gadjah Mada University, Yogyakarta, Indonesia \\ ${ }^{2}$ Department of Information Technology, Gadjah Mada University, Yogyakarta, Indonesia \\ ${ }^{3}$ Department of Information Technology, Gadjah Mada University, Yogyakarta, Indonesia \\ *Corresponding author.Email: gracedopi08@mail.ugm.ac.id
}

\begin{abstract}
This research was conducted using a literature review method to analyze various studies that will identify the type of analysis with the attributes used, the methods used, the methods most often used, and the methods that have the best performance. This study collected research from 2016 - July 2021, selected based on predetermined criteria and then collected 40 papers. This review found that there are four research topics, namely estimation, classification, clustering, and association. The findings of this study are four research topics, namely fundamentals, technicals, sentiment analysis, and even a combination of analyzes that use their respective attributes and datasets. There are thirty-one different methods found to be used in predicting stock prices. LSTM, MLP, RF, and SVM are the most widely used methods. In addition, MLP is a method that gives the best performance of 71.63\% and LSTM of $70 \%$. The use of combined machine learning methods with ensemble techniques, deep learning, and selection of input attributes in pre-processing is recommended for better accuracy.
\end{abstract}

Keywords: Systematic literature review, stock price prediction, technical analysis, fundamental analysis, sentiment analysis, machine learning, deep learning.

\section{INTRODUCTION}

Stock price analysis and prediction is the most challenging issue in financial matters [1]. That is because financial markets are very risky, chaotic, dynamic, and uncertain. Fundamental and Technical are the two main analyzes for making investment decisions in the stock market [2]. In addition to analyzing these forms, stock prices are influenced by many factors such as economic policy, current news, political events, and investor sentiment [3][4].

Each form of analysis has its attributes to analyze or predict stock prices. Technical analysis is an analytical methodology to predict prices through the study of past market data. Technical Analysis will use several attributes from historical market data such as date, open price, closing price, highest price, lowest price, volume, and other technical data. Fundamental analysis involves studying overall economic and industry conditions with the financial condition and quality of company management to predict price changes. Fundamental analysis will use fundamental data taken from the company. And sentiment analysis in the form of analysis of news sentiment, social media, and investor sentiment. In addition, a combination of the two attributes is also used, such as combining technical attributes and sentiment attributes. A combination of technical and fundamental attributes.

The COVID-19 pandemic has also affected the stock market and economic growth in Indonesia, as evidenced by the decline in stock prices in all industrial sectors. However, from this it was found that during the pandemic, stock investors increased. This increase is mostly due to fomo or just following the trend of stocks that have increased considerably during the pandemic. Many new stock investors who just because of fomo come into play without learning it first so they experience not small losses. So stock forecasting will help investors make profits and help the stock market recover due to the pandemic. 
Researchers use various methods and algorithms to find the best accuracy. For example, [12], [13], [9] use the Random Forest algorithm to predict stock prices or actions such as buy, sell, and hold. In addition, some researchers also use Deep Learning such as Artificial Neural Networks, LSTM, MLP in studying stock movements [14], [15], [16]. Furthermore, various other algorithms will be using.

In addition to methods and algorithms, it is also essential to know the current research trends, the type of analysis used, processing input attributes to get better accuracy.

Some researchers have reviewed the predictions related to stocks, such as research [17] in which the research only analyzes the technical analysis without considering the form of fundamental analysis and sentiment. This study was conducted to analyze research in 2010 - 2018 related to stock price predictions.

Therefore it is crucial to complement the previous research that has not been done. Such as the type of analysis that is not only centered on one type of analysis but combines types of analysis, in addition to previous studies that have not been complete researching the latest research. So this study focuses on researching research from 2016 - July 2021 to identify the type of research used, the research topic, the methods used, the most widely used method, and the method that has the best work performance. The following is the arrangement of writing in this paper. In part 2, the research methods used in this study will explain, part 3 will describe the research results, and part 4 will describe the conclusions and future research.

\section{RESEARCH METHODS}

Systematic Literature Review will be a reference in reviewing the literature on stock price predictions. System Literature Review defines as the process of identifying, assessing, and interpreting all available research evidence to provide answers to research questions [18]. Based on the rules in the systematic literature review, a step will carry out, namely, describing the research questions that are the research objectives so that the research is focused. Then explain the search strategy, including any data sources and databases, including the keywords used in the search. After that, select the data obtained based on predetermined criteria. Unsuitable studies will also be filtered. To make it easier for readers to understand, Figure 1 shows the research flow.

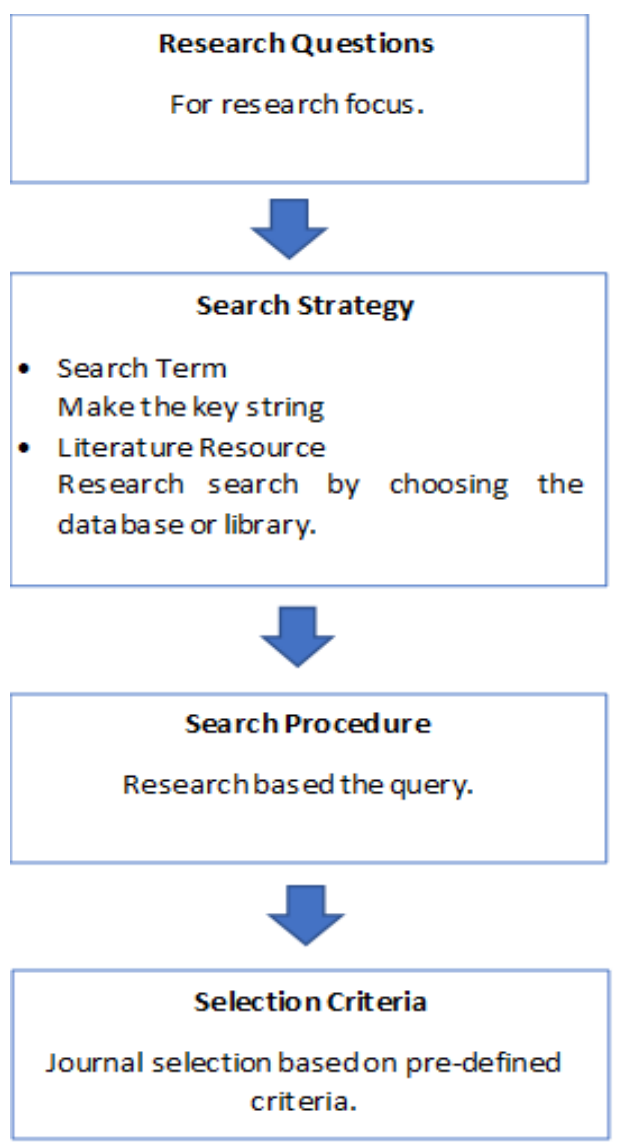

Figure 1. Research Flow.

\subsection{Research Questions}

In order for the research to remain focused, it is necessary to define the research questions. The Research Question design adopted the PICOC [18] Population, Intervention, Comparison, Results, and Context. Table 1 shows the PICOC structure of the research questions.

Table 1. $P I C O C$

\begin{tabular}{|l|l|}
\hline Structure & Description \\
\hline Population & Stock, Stock Market \\
\hline Intervention & $\begin{array}{l}\text { Stock Prediction, Stock Forecasting, } \\
\text { Technical Analysis, fundamental } \\
\text { analysis, sentiment analysis }\end{array}$ \\
\hline Comparison & n/a \\
\hline Outcomes & $\begin{array}{l}\text { Prediction accuracy of stock return, } \\
\text { successful stock prediction methods }\end{array}$ \\
\hline Context & Studies in stock forecast \\
\hline
\end{tabular}

Based on the PICOC table, research questions are makes of what will expect from this research. For this reason, the following research questions will formulate: 
RQ1: What are the trending research topics studied in the field in the field of stock prediction?

RQ2: What type of analysis is most widely used in predicting stocks?

RQ3: What are the methods used in predicting stocks?

RQ4: What are the most frequently used methods of predicting stocks?

RQ5: What is the best performing method for forecasting stocks?

\subsection{Search Strategy}

\subsubsection{Search Term}

To define a search string, we first define keywords with potential alternatives to ensure that the selected literature will be relevant to the study. In this SLR, we will only use articles that have writing in English. The keywords used to build the search query are as follows:

1) Stock market, Stock price, Stock index

2) Sentiment analysis, fundamental analysis, technical analysis

3) Forecasting, prediction

Once these search keywords are defined, a search query will create using a boolean operator for the next step. The search queries used are:

("Stock market" or "Stock price" or "Stock Index") AND ("sentimental analysis" or "fundamental analysis" or "technical analysis") AND (forecast* or predict $\left.{ }^{*}\right)$

\subsubsection{Literature Resource}

The search process in this study selects a digital library or database to review. This step recommends using a database or library that is relevant to the research used. The most popular digital libraries or databases will broaden the search and make it possible to find relevant articles. The following is a list of digital databases used to search for articles:

1) IEEE Explore (ieeexplore.ieee.org)

2) ScienceDirect (sciencedirect.com)

3) Springer (springerlink.com)

In the search for articles on Literature Source limiting research from 2016-2021.

\subsection{Search Procedure}

On selecting articles that will meet the requirements, the following process is followed by searching based on a query compiled from keywords and filtering the year and type of article. Then filtering is done by reviewing the title and abstract in order to remove irrelevant information easily. Then select all the literature based on the existing criteria and analyze the selected articles to find answers to research questions.

\subsection{Selection Criteria}

This stage is the filtering stage for excluding articles selected initially, which must match the expected criteria. The exclusion criteria are:

a. Research has no contribution to the field of machine learning or deep learning

b. Research does not display research results.

The selection of articles from beginning to end can be seen in Table 2, where the total data that came out according to the query were 2706 articles. Then the articles that download were 269 articles. After reviewing the exclusion criteria and the desired criteria, 40 articles were selected for further research.

Table 2. Search Result

\begin{tabular}{|l|c|c|}
\hline \multicolumn{1}{|c|}{ Portal } & $\begin{array}{c}\text { Result Based } \\
\text { on keywords }\end{array}$ & $\begin{array}{c}\text { Download } \\
\text { Journal }\end{array}$ \\
\hline Science Direct & 569 & 131 \\
\hline IEEE Explore & 117 & 59 \\
\hline Springer & 2020 & 269 \\
\hline Total & 2706 & 269 \\
\hline
\end{tabular}

\section{RESEARCH RESULT}

\subsection{The Most Trending Topics in the Field of Stock Price Prediction}

This research is to know what trending research topics studies in the field of stock prediction. The analysis carries out by classifying 41 selected articles based on the research topic. The research topics dividing into four topics:

1. Forecasting or forecasting or prediction.

In this topic, research carries out to estimate, predict, or predict stock price returns using forecasting or regression algorithms. 
2. Grouping

In this topic, the research implementation uses a classification algorithm to classify research into two or more classes such as "UP and Down," "Buy and Hold," or "Buy, Sell, Hold.

3. Clustering

In this topic, the research implementation uses a clustering algorithm in which stocks will be grouped based on investment decision-making.

\section{Association}

In this topic, research will find the relationship between stock price movements from one thing, for example, the relationship between the emergence of bullish and bearish signal indicators for stock price movements.

Estimates or forecasts or predictions is to predict stock prices using several algorithms using Artificial Neural Networks [19], [14] even using backpropagation artificial neural networks, [20] using MLP, [21] using RNN, and various other Neural Network methods. In addition, using regression, namely Lasso regression [22].

Then the grouping will give the output of the grouping of stock data such as "buy," "sell," "hold" [7], [12], [23], or "up" and "down" [24]. Several algorithms found will classify in this study, namely LSTM [10], [24]-[28], Random Forest used in research [9], [13], [29], and also random forest ensemble in research [12]. . Also using other algorithms such as fuzzy logic, MLP, FNN, and Decision Boosted Tree [2], [8], [23], [30]. Several studies have compared the best method for clustering, such as [31] comparing Naive Bayes and SVM, and found that Naive Bayes is better than others.

In clustering, the research [5] found using biclustering to find buying and selling patterns. In addition, research [32] conducted K-means to find indicators that affect market mood to find forecasting models for companies that develop for each market situation. Furthermore, the association method in research [33] also uses ANN Backpropagation to find the relationship between variables and stock prices on the following day.

Figure 2 shows the total distribution of stock prediction research topics from 2016 to June 2021. The most research topic is classification as much as $65 \%$, followed by estimation research topics as much as $28 \%$, after that clustering is $5 \%$, and association is the most little was done, by $2 \%$ of the total research.

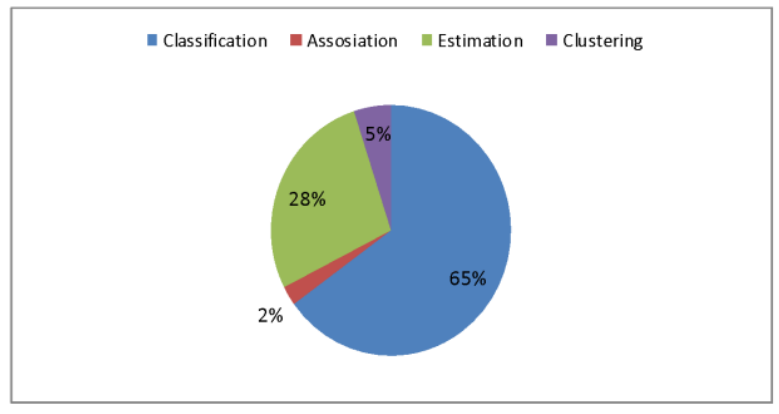

Figure 2. Research Topic Distribution

\subsection{The Most Type of Analysis Used in Predicting Stocks}

Several forms of analysis are carried out with different attributes in predicting stock prices. The three most core types of the analysis found in this study namely technical analysis, sentiment analysis, and fundamental analysis. Technical analysis uses historical stock market data or technical data used as attributes, which will then use to predict the stock market. The attributes used are different; some researchers use historical data directly, such as open prices, closing prices, volumes, etc. [6], [15], [19], [27], [33], [34]. In addition, some use attributes in the form of technical data such as Moving Average, Bollinger Bands, Weighted Moving Average (WMA) and share other technical data attributes [7], [12], [23], [29], [35]- [39]. Then sentiment analysis is carried out by analyzing data or sentiment from an object in the form of news and social media to predict stock prices. In research [9] conducted sentiment analysis using Twitter data with tweet and date indicators.

Furthermore, fundamental analysis is an analysis that uses a company's financial data to look at the financial health of the company, the attributes that exist also vary. This literature review uses fundamental analysis with 21 fundamental attributes to predict stock prices.

In addition, it is possible to use more than one form of analysis, in other words combining two or more forms of analysis above to predict stocks. Sentiment and technical analysis mean using technical and sentiment attributes to predict stocks; the attributes used are different. As done by [1], [10], [20], [21], [24], [25], [30], [31], [40], [41] using a combination of technical attributes and analysis sentiment to be an attribute of predicting stock prices. Then also several studies using a combination of technical and fundamental analysis in which technically and fundamental attributes company's used to predict stock prices [2], [11], [13], [32], [42], [43]. Attributes of 
technical and fundamental are different from each researcher. The use of fundamental attributes and other sentiments not found in this experiment, also includes using the third form of analysis at once. However, a study conducted by [44] suggested increasing the attribute fundamental to complement the research done with the attributes of the technical and sentiment.

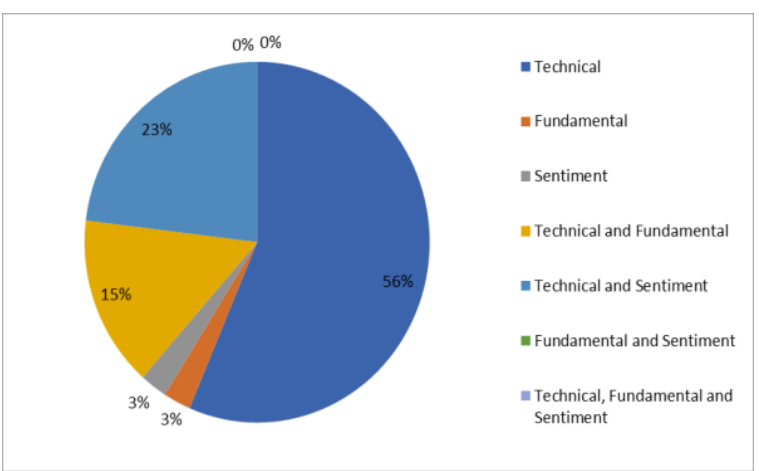

Figure 3. Analysis Type Distribution

Figure 3 displays the distribution of this paper where the use of technical attributes is the most commonly found in predicting the stock price by $56 \%$. The combined use of technical attributes and other sentiments by $23 \%$, the combined use of fundamental and technical attributes by $15 \%$, the use of fundamental attributes by $3 \%$, sentiment analysis by $3 \%$, and there has been no combined use of fundamental attributes and sentiment analysis, and the use of all three at once.

Each type of analysis sometimes has a different process, such as research conducted by [15] which performs technical analysis using attributes of past stock data and RSI values. This study describes the study in 6 steps that is presented in Figure 4. The share price used in this study is from finance.yahoo.com for training and research purposes, where the data taken for training is stock price data between $1 / 1 / 1997$ to
$31 / 12 / 2006$, then for stock price test data between $1 / 1 / 2007$ to $1 / 1 / 2017$. In phase 0 , the downloaded stock price is normalized based on the closing price as in the picture. Then in phase 1 , RSI values in different intervals are between 1 to 20 days, and the SMA for an interval between 50-200 days calculating to determine the uptrend or down. The data is computing by adopting the TA4J2 Technical Analysis for the Java library. Algorithm Genetic is used in phase 2 to find the best RSI values for buying and selling points of previous uptrends and downs found in randomly initialized data populations that will then be input in phase 3 using MLP. Phase 3 will process inputs into training and testing data sets using Apache Spark MLib. Phase 4 will do voting to find the best value used as a prediction of RSI intervals. The predicted results of the classifications of "sell," "buy," and "hold" will be calculated, which has several more than 14 , and the result will be a reference for predictions. Then phase 5 is the evaluation of stock prices based on predictions.

Furthermore, the research was conducted by [46] using fundamental analysis. First, make the selection of a company based on its liquidity. A total of 15 companies applied to train models. The financial data is used as a training model and test model. Training data was used from $18 / 02 / 2019$ to $01 / 09 / 0219$, while test data was between $1 / 10 / 2019$ to $15 / 12 / 2019$. The model trains using fundamental attributes: Volume, History, Expiry, Take Profit, Stop Loss, Minimum, RSI, Donchian channel, MACD, MFI, and Moving average. Then, various combinations of parameters checking to get the best results by removing, adding, and crossing them. At first, the basic parameters are analyzed to determine which works best (history, expiration, minimum), then other parameters are added and crossed. The results compare by using metrics such as precision, recall, f1, portfolio results, and transaction number.

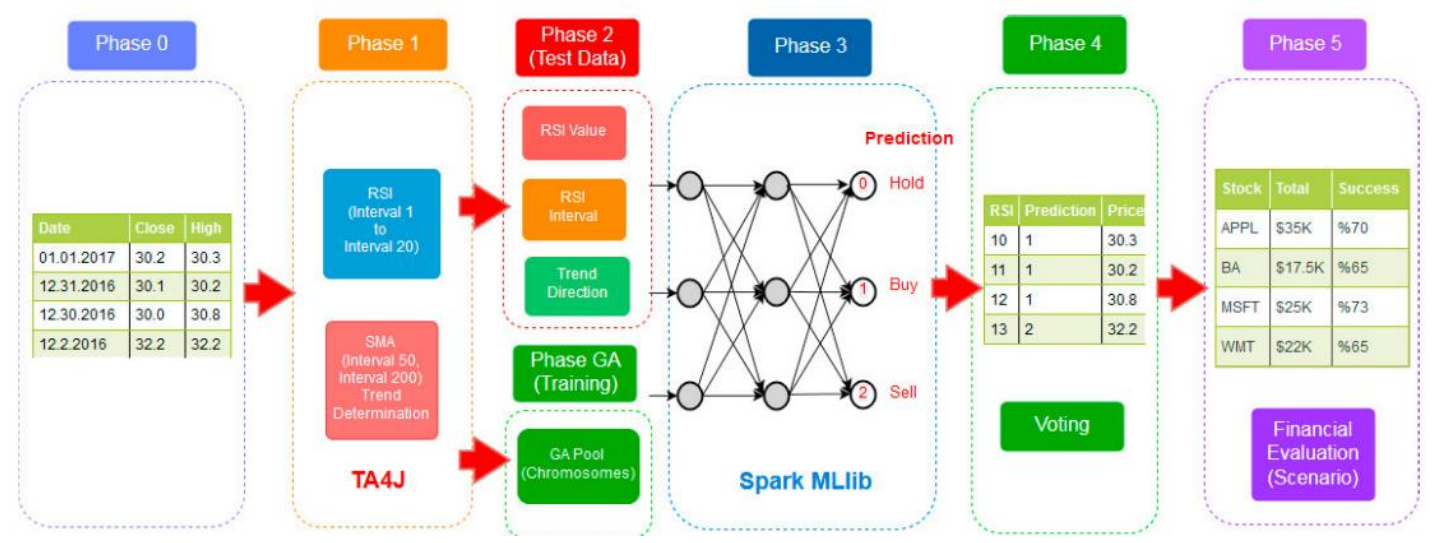

Figure 4. Proposed Method (Genetic Algorithm and MLP) [15]. 


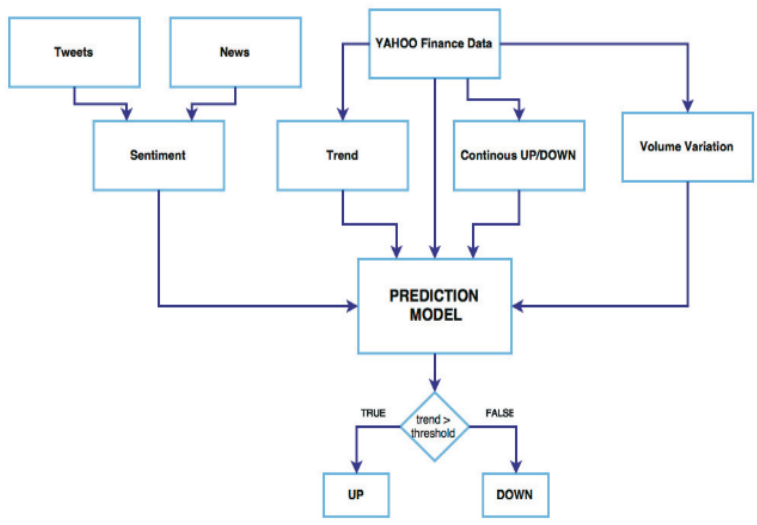

Figure 5. Prediction Model for Daily Prediction Model [30]

Research [30] conducts research combining sentiment and technical analysis. This research step image can be seen in Figure 5. First, Twitter and news data will use as input data for sentiment research, then the history of past data is used to see trends, continuous up/down, volume variation. Some of these inputs will be combined into the prediction model using machine learning. The results of the study will produce an output in the form of up or down.

Then on research [2] using a combination of forms of fundamental and technical analysis. The attribute used for fundamental analysis is the financial ratio performing the feature selection and discretization data. While in technical analysis will use stock prices that initially done discretization data then will do technical analysis. The output of the two previous analyses will be the input of the Hybrid Model to enter the prediction system. Data collection obtained from the Nasdaq Stock Market will be input along with the output of the previous Hybrid Model that will be processed using MLP to display accuracy.

\subsection{Method Used in Predicting Stock}

In predicting stocks, various machine learning methods are using to research the possibility of finding the best method of predicting stock prices. Research gathered from 2016-July 2021 found as many as 31 algorithms in adoption to find the best accuracy. Figure 6 displays all methods used.

\subsection{The Most Used Methods in Stock Prediction}

Based on Figure 6, we can see that the most widely used methods are MLP and LSTM, which Random Forest and Support Vector Machine follow. Therefore, this method still has the potential in the future to be used to predict stock prices.

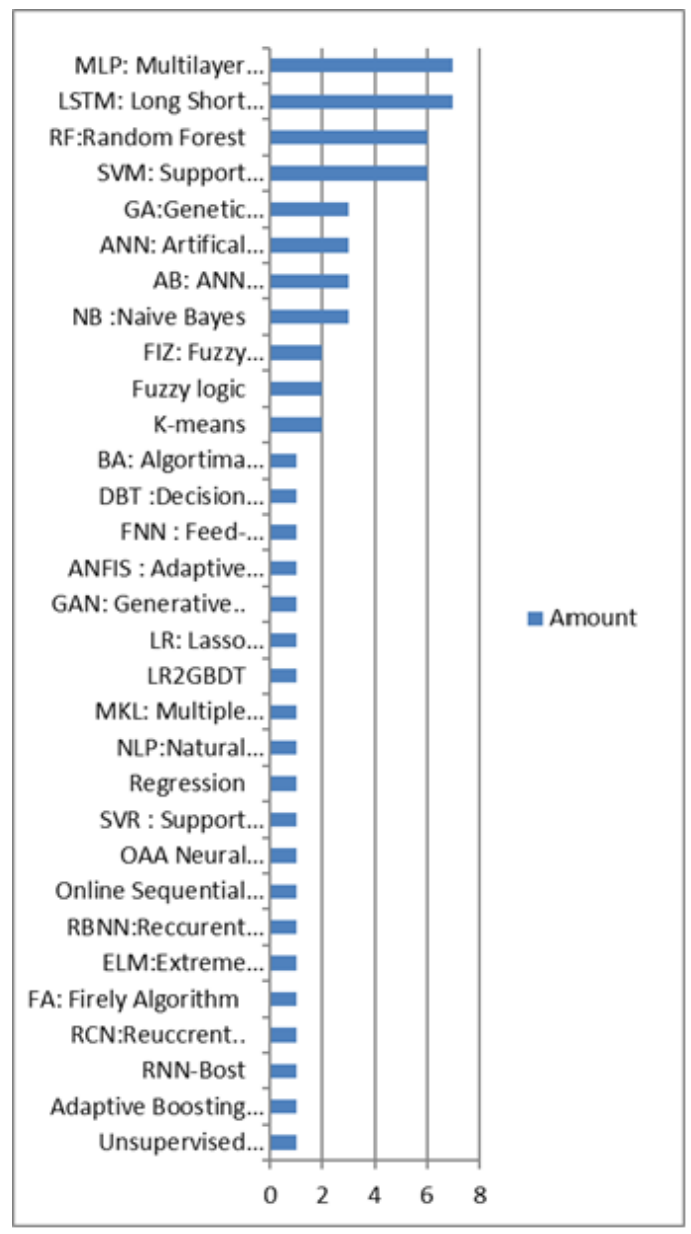

Figure 6. Method used in stock prediction

\subsection{The Best Performing Methods in Stock Prediction}

Various methods are using to get better accuracy in predict stocks. Although using the same process, the prediction results remain different depending on the dataset used and the type of attributes used. These studies often lay out the results without providing research accuracy, so only a few studies can be summarized.

In Table 3, the study results explained the accuracy value, and it is found that the MLP managed to predict the stock price with an accuracy of $71.63 \%$ using technical analysis [15]. Research[15] shows results that trading systems produce to buy, sell and hold classification results that are comparable or better when compared to Buy and control, then can use for various stock markets even for more extended periods. Then ANN Backpropagation generated RMSE 0.0348 in its research in analyzing stock prices using technical analysis [32]. LSTM in predicting stocks using technical analysis and sentiment analysis from Twitter 
data with $70 \%$ accuracy and then random forest in fundamental analysis has an accuracy of $66.30 \%$.

Table 3. Method with best performing

\begin{tabular}{|c|c|c|c|c|}
\hline Author & Method & $\begin{array}{c}\text { Analysis } \\
\text { Type }\end{array}$ & $\begin{array}{c}\text { Accu- } \\
\text { racy }\end{array}$ & RMSE \\
\hline$[15]$ & MLP & Technical & $71.63 \%$ & - \\
\hline$[33]$ & $\begin{array}{c}\text { ANN } \\
\text { Backpro } \\
\text { pogation }\end{array}$ & Technical & - & 0.0348 \\
\hline$[24]$ & LSTM & $\begin{array}{c}\text { Technical } \\
\text { and } \\
\text { Sentiment } \\
\text { (Twitter) }\end{array}$ & $70 \%$ & - \\
\hline$[13]$ & $\begin{array}{c}\text { Random } \\
\text { Forest }\end{array}$ & $\begin{array}{c}\text { Fundamental } \\
\text { nof }\end{array}$ & $66.30 \%$ & - \\
\hline
\end{tabular}

The method with the best performance is also a list of the most frequent methods used, so using some of these methods is still very complicated for further research. The methods proposed for use in subsequent research are the LSTM and MLP methods. However, it is necessary to re-research using the same data to see which one is the best of these algorithms.

Based on research, some weaknesses that cause accuracy is not maximal are: first: do a thorough analysis of the state of the market, such as conducting a combination of forms of research to get better accuracy. Then pre-processing is needed, such as attribute selection to get the best accuracy, so it is better to do feature selection first for each attribute to be inputted. Then, try to use various possible algorithms or hybrids to find what is the most appropriate method to use in predicting stocks.

It is proposed to perform a combination of types of analysis. In addition, pay more attention to preprocessing processes such as feature selection of input data to be used. In this study, it was found that he use of deep learning was also found to provide improved accuracy.

\section{CONCLUSION}

This literature review authenticates and analyzes the trends of research topics, the types of analysis used, the methods used, the most widely used methods, and the methods that perform best. This literature review adopts[17] in research methods. A total of 40 studies from 2016 to June 2021, taking into account expected research criteria. In this review, four research focuses find estimation, classification, clustering, and association. It found that category became the most widely used research topic at $65 \%$ of studies, followed by an estimate by $28 \%$, then clustering by $5 \%$ and association of $2 \%$ of the total distribution.

Then also researched the type of analytical techniques used, namely technical analysis, fundamental analysis, sentiment analysis, or a combination of 2 or more analyses. Technical analysis uses data sets from historical stock data, fundamental analysis using data sets derived from company fundamentals, and sentiment analysis, namely public sentiment related to the stock can obtain from the news or social media. It is also used in a combination of both forms of analysis. From the results of the total distribution of this literature review, it was founded that $56 \%$ of research uses technical analysis and technical attributes or datasets in their research, then $23 \%$ combined from technical analysis and sentiment analysis. Combined fundamental and technical by $15 \%$, fundamental analysis by $3 \%$, sentiment analysis by $3 \%$, no research found using combined sentiment analysis and fundamental analysis, and three forms of analysis.

This review found 31 algorithmic methods used in this study. MPL and LSTM became the dominant methods used, then RF and SVM. To determine performance methods cannot be chosen due to different data sets and attributes; still, it is understood that MPL can provide an accuracy of $71.63 \%$ in detecting stock prices using this type of technical analysis [15]. Additionally, LSTM provides an accuracy performance of $70 \%$ in technical analysis and sentiment [23]. The method will produce good performance with suitable techniques and proper preprocessing for each data set.

In the future, this systematic literature review proposes to perform a combination of types of analysis. And also, using combined machine learning methods through ensemble techniques, using deep learning methods is expected to give better results. Processing the selection of influential input attributes is also essential for further research.

\section{REFERENCES}

[1] G. Attanasio, L. Cagliero, P. Garza, and E. Baralis, "Combining news sentiment and technical analysis to predict stock trend reversal," in Combining news sentiment and technical analysis to predict stock trend reversal, Nov 2019, vol. 2019-Novem, pp. 514-521, doi: 10.1109/ICDMW.2019.00079.

[2] A. Namdari and Z. S. Li, "Integrating Fundamental and Technical Analysis of Stock 
Market through Multi-layer Perceptron," Okt 2018, doi: 10.1109/TEMSCON.2018.8488440.

[3] C. Zhang, Z. Ji, J. Zhang, Y. Wang, X. Zhao, and Y. Yang, "Predicting Chinese stock market price trend using machine learning approach," ACM Int. Conf. Proceeding Ser., pp. 6-9, 2018, doi: 10.1145/3207677.3277966.

[4] S. Kumar and D. Ningombam, "Short-Term Forecasting of Stock Prices Using Long Short Term Memory," in Proceedings - 2018 International Conference on Information Technology, ICIT 2018, Des 2018, pp. 182-186, doi: 10.1109/ICIT.2018.00046.

[5] J. Yang and Q. Huang, "Automated trading based on biclustering mining and fuzzy modeling," in ICARM 2016 - 2016 International Conference on Advanced Robotics and Mechatronics, Okt 2016, pp. 591-596, doi: 10.1109/ICARM.2016.7606987.

[6] F. Zhou, Q. Zhang, D. Sornette, and L. Jiang, "Cascading logistic regression onto gradient boosted decision trees for forecasting and trading stock indices," Appl. Soft Comput. J., vol. 84, Nov 2019, doi: 10.1016/j.asoc.2019.105747.

[7] S. Boonpeng and P. Jeatrakul, "Decision support system for investing in stock market by using OAA-Neural Network," in Proceedings of the 8th International Conference on Advanced Computational Intelligence, ICACI 2016, Apr 2016, pp. 1-6, doi: 10.1109/ICACI.2016.7449794.

[8] Y. Huang, L. Fernando Capretz, and D. Ho, "Neural Network Models for Stock Selection Based on Fundamental Analysis," 2019.

[9] N. N. Reddy, N. E, and V. Kumar, "Predicting Stock Price Using Sentimental Analysis Through Twitter Data," Proc. IEEE Conecct 2020 6th Int. Conf. Electron. Comput. Commun. Technol. July 2-4, 2020, 2020.

[10] K. M, K. J, E. R. T, and A. S, "Stock Market Prediction with Historical Time Series Data and Sentimental Analysis of Social Media Data," Proc. Int. Conf. Intell. Comput. Control Syst., 2020.

[11] E. Beyaz, F. Tekiner, X. J. Zeng, and J. Keane, "Comparing Technical and Fundamental Indicators in Stock Price Forecasting," in
Proceedings - 20th International Conference on High Performance Computing and Communications, 16th International Conference on Smart City and 4th International Conference on Data Science and Systems, HPCC/SmartCity/DSS 2018, Jan 2019, pp. 1607-1613, doi: 10.1109/HPCC/SmartCity/DSS.2018.00262.

[12] IEEE Computational Intelligence Society, Institute of Electrical and Electronics Engineers, and B. C. IEEE World Congress on Computational Intelligence (2016: Vancouver, "Equity Price Direction Prediction For Day Trading Ensemble Classification Using Technical Analysis Indicators With Interaction Effects," IEEE Comput. Intell. Soc. Inst. Electr. Electron. Eng. IEEE World Congr. Comput. Intell. (2016 Vancouver, B.C.), 2016.

[13] L. S, "Impact of Financial Ratios and Technical Analysis on Stock Price Prediction Using Random Forests," Ethical Integr. Comput. Drone Technol. Humanit. Sustain. 9th-11th Nov. 2017, Kuching, Sarawak, Malaysia, 2017.

[14] P.-C. Lan, W.-L. Kung, Y.-L. Ou, C.-Y. Lin, C. H. Hu, and Y.-H. Wang, "Machine learning model with technical analysis for stock price prediction: Empirical study of Semiconductor Company in Taiwan," 2019 Int. Symp. Intell. Signal Process. Commun. Syst. (ISPACS 2019) Proc. Dec. 3-6, 2019, Taipei, Taiwan., 2019.

[15] O. B. Sezer, M. Ozbayoglu, and E. Dogdu, “A Deep Neural-Network Based Stock Trading System Based on Evolutionary Optimized Technical Analysis Parameters," in Procedia Computer Science, 2017, vol. 114, pp. 473-480, doi: 10.1016/j.procs.2017.09.031.

[16] A. Sarkar, A. K. Sahoo, S. Sah, and C. Pradhan, "LSTMSA: A Novel Approach for Stock Market Prediction Using LSTM and Sentiment Analysis," 2020.

[17] D. P. Gandhmal and K. Kumar, "Systematic analysis and review of stock market prediction techniques," Comput. Sci. Rev., vol. 34, pp. 100190, 2019, doi: 10.1016/j.cosrev.2019.08.001.

[18] B. Kitchenham and S. Charters, "Guidelines for performing systematic literature reviews in software engineering," Tech. report, Ver. 2.3 
EBSE Tech. Report. EBSE, 2007.

[19] Y.-L. Cai, K. Kannan, Y.-H. Xie, and L. Zhao, "E-Commerce: Stock Market Analysis Blended With Mining and Ann," 2019 IEEE Int. Conf. Ind. Eng. Eng. Manag., 2019.

[20] X. Zhang, J. Shi, D. Wang, and B. Fang, "Exploiting investors social network for stock prediction in China's market," J. Comput. Sci., vol. 28, pp. 294-303, Sep 2018, doi: 10.1016/j.jocs.2017.10.013.

[21] W. Chen, C. K. Yeo, C. T. Lau, and B. S. Lee, "Leveraging social media news to predict stock index movement using RNN-boost," Data Knowl. Eng., vol. 118, no. December 2017, pp. 14-24, 2018, doi: 10.1016/j.datak.2018.08.003.

[22] A. Rastogi, A. Qais, A. Saxena, and D. Sinha, "Stock Market Prediction with Lasso Regression using Technical Analysis and Time Lag," Apr 2021, doi: 10.1109/I2CT51068.2021.9417935

[23] S. Lauguico, R. Concepcion, J. Alejandrino, D. Macasaet, R. R. Tobias, and E. Bandala, "A Fuzzy Logic-Based Stock Market Trading Algorithm Using Bollinger Bands," 2019 IEEE 11th Int. Conf. Humanoid, Nanotechnology, Inf. Technol. Commun. Control. Environ. Manag. ( HNICEM )., 2019.

[24] V. Sharma, R. Khemnar, R. Kumari, and D. B. R. Mohan, "Time Series with Sentiment Analysis for Stock Price Prediction," 2019 2nd Int. Conf. Intell. Commun. Comput. Tech. Manipal Univ. Jaipur, Sep. 28-29, 2019.

[25] X. Li, P. Wu, and W. Wang, "Incorporating stock prices and news sentiments for stock market prediction: A case of Hong Kong," Inf. Process. Manag., vol. 57, no. 5, Sep 2020, doi: 10.1016/j.ipm.2020.102212.

[26] Z. Lanbouri and S. Achchab, "Stock market prediction on high frequency data using longshort term memory," in Procedia Computer Science, 2020, vol. 175, pp. 603-608, doi: 10.1016/j.procs.2020.07.087.

[27] D. M. Q. Nelson, A. C. M. Pereira, and R. A. De Oliveira, "Stock market's price movement prediction with LSTM neural networks," in Proceedings of the International Joint Conference on Neural Networks, Jun 2017, vol. 2017-May, pp. 1419-1426, doi:

\subsection{9/IJCNN.2017.7966019.}

[28] K. Khare, O. Darekar, P. Gupta, and D. V. Z. Attar, "Short Term Stock Price Prediction Using Deep Learning," RTEICT-2017 2nd IEEE Int. Conf. Recent Trends Electron. Inf. Commun. Technol. Proc. 19-20 May 2017, 2017.

[29] H. Kim and S. T. Han, "The Enhanced Classification for the Stock Index Prediction," in Procedia Computer Science, 2016, vol. 91, pp. 284-286, doi: 10.1016/j.procs.2016.07.077.

[30] A. Nayak, M. M. M. Pai, and R. M. Pai, "Prediction Models for Indian Stock Market," in Procedia Computer Science, 2016, vol. 89, pp. 441-449, doi: 10.1016/j.procs.2016.06.096.

[31] G. Jariwala, H. Agarwal, and V. Jadhav, "Sentimental Analysis of News Headlines for Stock Market," Nov 2020, doi: 10.1109/INOCON50539.2020.9298333.

[32] E. Beyaz, F. Tekiner, X. J. Zeng, and J. Keane, "Stock Price Forecasting Incorporating Market State," in Proceedings - 20th International Conference on High Performance Computing and Communications, 16th International Conference on Smart City and 4th International Conference on Data Science and Systems, HPCC/SmartCity/DSS 2018, Jan 2019, pp. 1614-1619, doi: 10.1109/HPCC/SmartCity/DSS.2018.00263.

[33] Universitas Telkom, Multimedia University, M. IEEE Systems, and Institute of Electrical and Electronics Engineers, Prediction of Agriculture and Mining Stock Value Listed in Kompas 100 Index Using Artificial Neural Network Backpropagation.

[34] N. Sirimevan, I. G. U. H. Mamalgaha, C. Jayasekara, Y. S. Mayuran, and C. Jayawardena, "Stock Market Prediction Using Machine Learning Techniques," 2019 Int. Conf. Adv. Comput. ICAC 2019, no. November, pp. 192197, 2019, doi: 10.1109/ICAC49085.2019.9103381.

[35] G. Jaiwang and P. Jeatrakul, "Enhancing support vector machine model for stock trading using optimization techniques," in 3rd International Conference on Digital Arts, Media and Technology, ICDAMT 2018, Jun 2018, pp. 2328, doi: 10.1109/ICDAMT.2018.8376489. 
[36] S. Dinesh, R. Nithin Rao, S. P. Anusha, and R. Samhitha, "Prediction of Trends in Stock Market using Moving Averages and Machine Learning," Apr 2021, doi: 10.1109/I2CT51068.2021.9418097.

[37] S. R. Das, D. Mishra, and M. Rout, "Stock market prediction using Firefly algorithm with evolutionary framework optimized feature reduction for OSELM method," Expert Syst. with Appl. X, vol. 4, pp. 100016, 2019, doi: 10.1016/j.eswax.2019.100016.

[38] X. and Zhang, A. Li, and R. Pan, "Stock trend prediction based on a new status box method and AdaBoost probabilistic support vector machine," Appl. Soft Comput. J., vol. 49, pp. 385-398, Des 2016, doi: 10.1016/j.asoc.2016.08.026.

[39] D. Zhang and S. Lou, “The application research of neural network and BP algorithm in stock price pattern classification and prediction," Futur. Gener. Comput. Syst., vol. 115, pp. 872879, Feb 2021, doi: 10.1016/j.future.2020.10.009.

[40] N. Kumar, R. Chauhan, and G. Dubey, "Applicability of Financial System Using Deep Learning Techniques," in Advances in Intelligent Systems and Computing, 2020, vol. 1097, pp. 135-146, doi: 10.1007/978-981-15-1518-7_11.

[41] A. Yadav, C. K. Jha, A. Sharan, and V. Vaish, "Sentiment analysis of financial news using unsupervised approach," in Procedia Computer Science, 2020, vol. 167, pp. 589-598, doi: 10.1016/j.procs.2020.03.325.

[42] K. Zhang, G. Zhong, J. Dong, S. Wang, and Y. Wang, "Stock Market Prediction Based on Generative Adversarial Network," in Procedia Computer Science, 2019, vol. 147, pp. 400-406, doi: 10.1016/j.procs.2019.01.256.

[43] M. Ahmad, H. Soeparno, and T. A. Napitupulu, "Stock trading alert: With fuzzy knowledgebased systems and technical analysis," in 2020 International Conference on Information Technology Systems and Innovation, ICITSI 2020 - Proceedings, Okt 2020, pp. 155-160, doi: 10.1109/ICITSI50517.2020.9264914.

[44] C.-Y. Lee and W. Soo, "Predict Stock Price with Financial News Based on Recurrent Convolutional Neural Networks."
[45] S. Ravikumar and P. Saraf, "Prediction of stock prices using machine learning (regression, classification) Algorithms," 2020 Int. Conf. Emerg. Technol. INCET 2020, pp. 1-5, 2020, doi: 10.1109/INCET49848.2020.9154061.

[46] K. Kaczmarczyk and M. Hernes, "Financial decisions support using the supervised learning method based on random forests," Procedia Comput. Sci., vol. 176, pp. 2802-2811, 2020, doi: 10.1016/j.procs.2020.09.276. 